\title{
Masa interauricular
}

Irene Juanes Domínguez

María Garrido Uriarte

Olatz Zaldua Irastorza

Servicio de Cardiología. Hospital Universitario Araba. Vitoria-Gasteiz. País Vasco. España

\section{Recibido: 22/07/2019}

Aceptado: 04/01/2020

En línea: 02/03/2020
Marta Torres Fernández

Ángel María Alonso Gómez

\section{Correspondencia}

Irene Juanes Domínguez irenejuanesdo@gmail.com

Citar como: Juanes-Domínguez I, Garrido-Uriarte M, Zaldua-Irastorza O, Torres-Fernández M, Alonso-Gómez, AM. Masa interauricular. Rev Ecocar Pract (RETIC). 2020 (Mar); 3 (1): 31-34. doi: 10.37615/retic.v3n1a8.

Cite this as: Juanes-Domínguez I, Garrido-Uriarte M, Zaldua-Irastorza O, Torres-Fernández M, Alonso-Gómez, AM. Interatrial mass. Rev Ecocar Pract (RETIC). 2020 (Mar); 3 (1): 31-34. doi: 10.37615/retic.v3n1a8.

\section{Palabras clave}

$\triangleright$ Masa auricular

$\triangleright$ Hematoma intramural

\section{Keywords}

$\triangleright$ Auricularmass

$\triangleright$ Intramural hematoma

\section{RESUMEN}

Se presenta el caso de una paciente con síndrome coronario agudo con elevación del ST que presentó disección y rotura de la arteria circunfleja. En el control tras el cateterismo se objetivó mediante ecocardiografía una masa redondeada hiperecogénica, de densidad homogénea en aurícula izquierda adherida al techo auricular que se confirmó por tomografía computarizada con contraste, describiéndose así mismo compresión de la vena pulmonar izquierda. El diagnóstico de hematoma intramural se confirmó mediante intervencionismo quirúrgico donde se procedió a la extracción del hematoma y de la sangre retenida.

ABSTRACT
We report a case of a patient with ST-elevation myocardial infarction with dissection and rupture of the circumflex
artery. Echocardiography after coronary angiography revealed a hyperechogenic rounded mass of homogeneous
density in left atrium that was confirmed by thoracic contrast-enhanced computed tomography, describing as
well compression of the left pulmonary vein. Intramural hematoma diagnosis was confirmed by surgical interven-
tion where extraction of retained blood was carried out.

\section{Presentación del caso}

Mujer de 47 años con factores de riesgo cardiovascular, fumadora e hiperlipidemia de reciente diagnóstico, sin otros antecedentes de interés. Es atendida en el servicio de Urgencias de un hospital secundario, a la media hora de inicio de dolor centrotorácico irradiado a brazo izquierdo. En el electrocardiograma realizado se observa una elevación del segmento ST en derivaciones inferiores (Figura 1).

Con el diagnóstico de síndrome coronario agudo con elevación del ST se activa el código infarto y se traslada a la paciente para la realización de cateterismo urgente a un hospital terciario de referencia. Se realiza cateterismo urgente con hallazgo de enfermedad coronaria de dos vasos: lesión del 75\% en coronaria derecha proximal y oclusión completa de la arteria circunfleja distal, con hematoma mural/disección coronaria espontánea como causante del infarto. Tras el inflado de balón de angioplastia en la arteria circunfleja, se visualiza rotura con fuga de contraste a nivel distal del segmento, por lo que se posiciona un stent recubierto en la zona de la fuga y se trata el resto de la lesión con un segundo stent también farmacoactivo. Se consiguió la desaparición completa de la fuga coronaria y la normalización del flujo coronario. Al terminar el procedimiento no se visualiza la presencia de derrame pericárdico mediante ecocardioscopia.
A su llegada al servicio de Medicina Intensiva la paciente se encuentra hemodinámicamente estable. Durante las siguientes horas al procedimiento presenta molestia torácica tipo opresiva acompañada de disnea sin taquipnea, ni desaturación que mejora con morfina y ansiolíticos. En la ecocardioscopia se describe una función ventricular conservada (FEVI) con acinesia del septoinferior e inferolateral basal y mínimo derrame pericárdico, con presencia de aumento de densidad ecogénica en aurícula izquierda; ¿ecocontraste espontáneo?

A las 32 horas del procedimiento persiste la sensación de molestia torácica y disnea sin alteraciones hemodinámicas, por lo que se repite la ecografía objetivando una masa redondeada hiperecogénica, con densidad homogénea en aurícula izquierda, no calcificada, adherida al techo auricular y con mínima separación de ambas hojas pericárdicas (Vídeo 1 y Vídeo 2). Se decide realizar una TC con contraste describiéndose un hematoma retroauricular izquierdo ${ }^{(1)}$ de $7 \times 6 \times 3,3 \mathrm{~cm}$ con compresión de la aurícula y del ostium de la vena pulmonar izquierda. En el interior del hematoma se visualiza en su porción más declive extravasación del contraste endovenoso, posiblemente por la presencia de sangrado activo probablemente a partir de la arteria circunfleja o, como alternativa diagnóstica, contraste retenido extravasado en el cateterismo (Figura 2).

Ante los hallazgos descritos se contacta con el servicio de Cirugía cardíaca y se decide traslado urgente a dicho centro para intervención urgente. Se realiza 
un orificio en el epicardio de la aurícula izquierda drenando el hematoma y la sangre retenida.

Tras el alta con betabloqueantes y doble antiagregación se realiza ecocardiografía de control al mes donde presenta una fracción de eyección del 43\% con un ventrículo ligeramente dilatado y acinesia inferior basal y media e hipocinesia severa inferolateral basal y medial. La cavidad auricular en los planos apicales de cuatro y dos cámaras se encuentra libre de masas (Vídeo 3). En plano apical de tres y cinco cámaras se aprecia una imagen adosada a la pared aurícula izquierda como probable secuela del proceso previo (Vídeo 4 y Vídeo 5, círculo amarillo).

\section{Estudio por imagen}

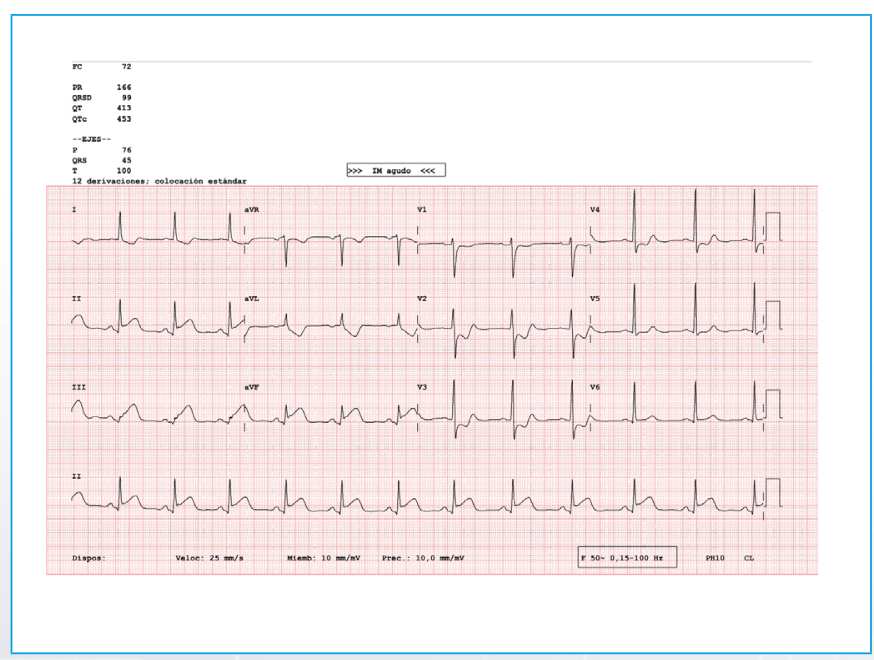

Figura 1. Ritmo sinusal. Elevación del segmento ST (lesión subepicárdica) en derivaciones inferiores inferiores: II, III y aVF. Infradesnivel "especular" del segmento ST (lesión subendocárdica) en I, aVL, V2, V3 y V4

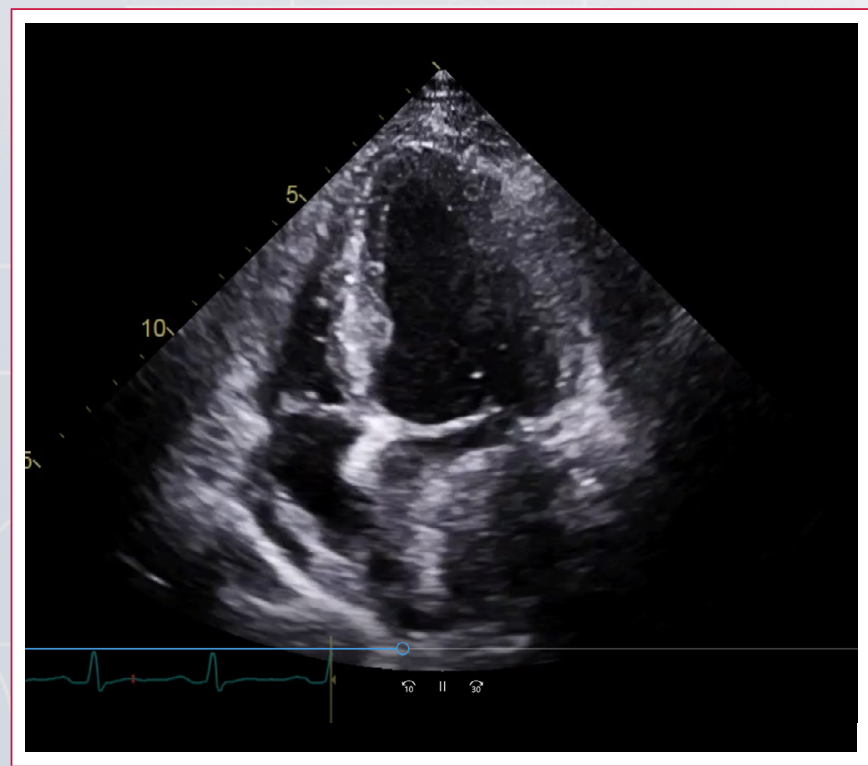

Vídeo 1. Plano apical de cuatro cámaras. Masa redondeada hiperecogénica, con densidad homogénea en aurícula izquierda. Mínima separación de hojas pericárdicas en la pared posterior de la aurícula derecha

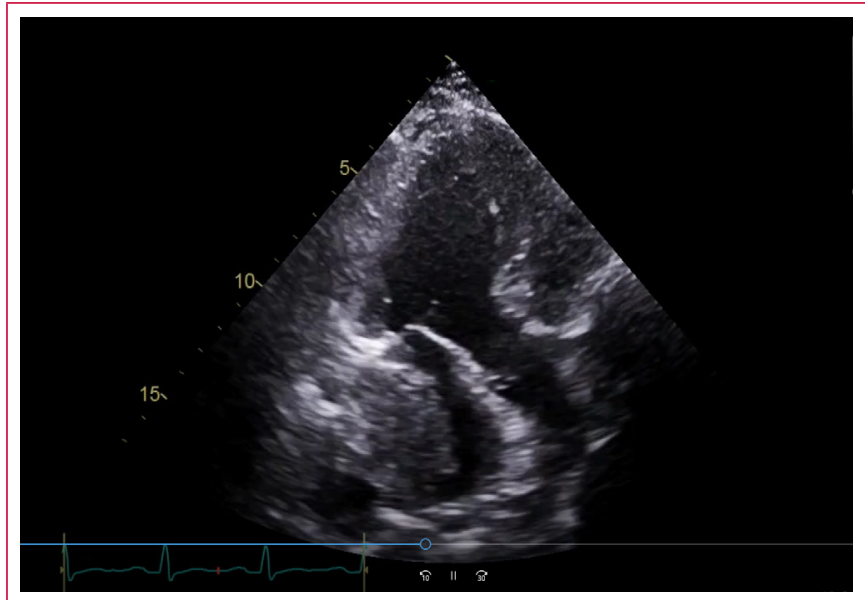

Vídeo 2. Plano apical de tres cámaras. Masa redondeada hiperecogénica con densidad homogénea en aurícula izquierda. Acinesia de los segmentos inferolateral basal y medio

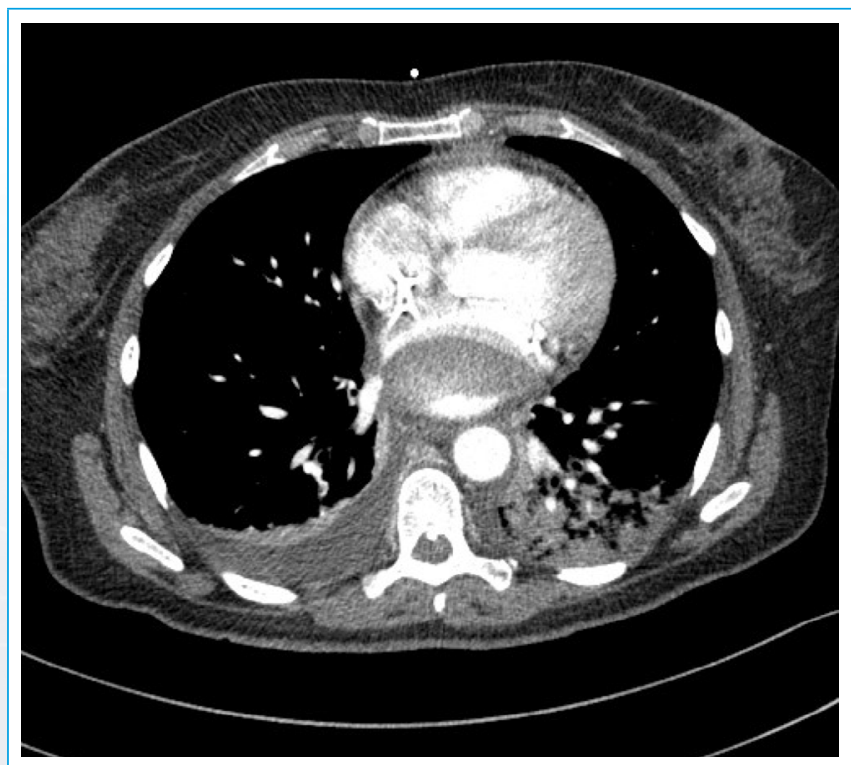

Figura 2. TC corte axial: hematoma retroauricular izquierdo de $7 \times 6 \times 3,3$ $\mathrm{cm}$ con compresión de aurícula y del ostium de la vena pulmonar izquierda (véase texto)

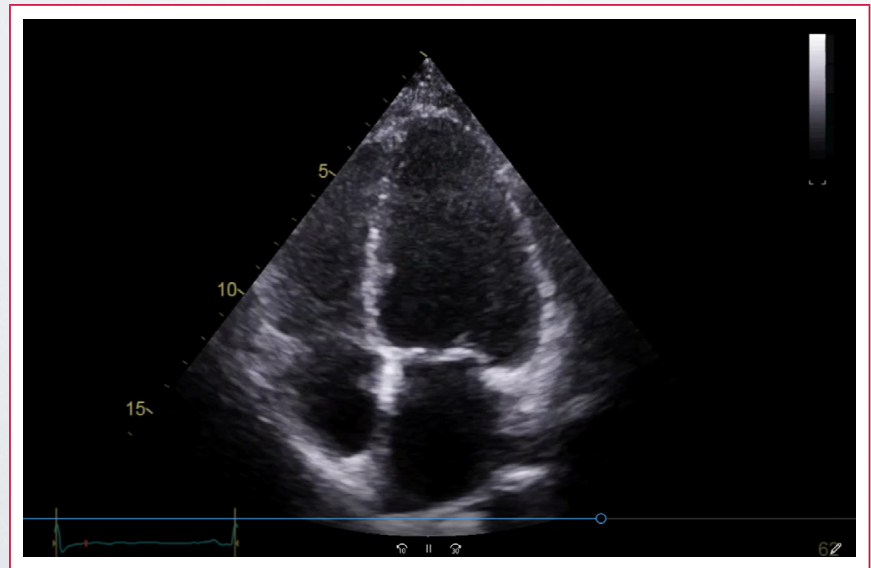

Vídeo 3. Plano apical de cuatro cámaras 


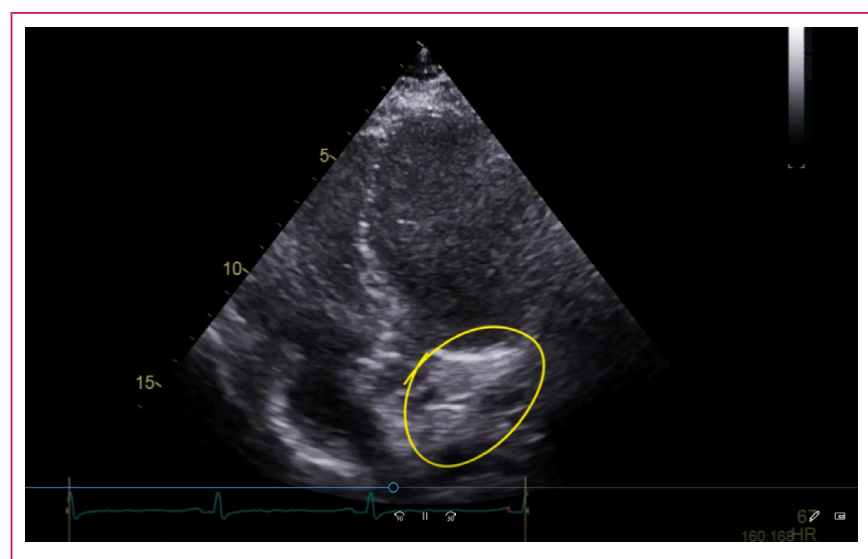

Vídeo 4. Plano apical de cinco cámaras (círculo amarillo: secuela posquirúrgica)

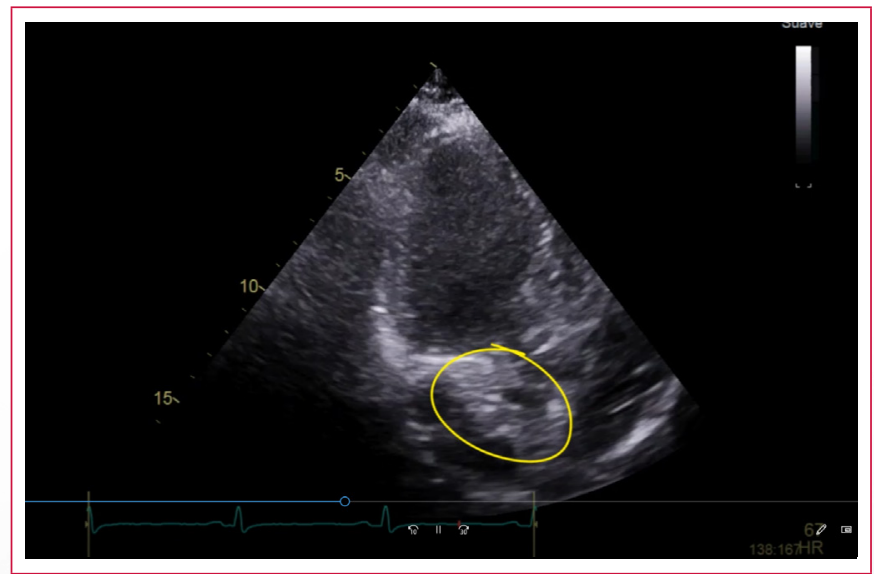

Vídeo 5. Plano apical de tres cámaras (círculo amarillo: secuela posquirúrgica)

\section{Discusión}

La presencia de una masa ocupante de espacio en la pared auricular izquierda tras intervencionismo coronario es un hallazgo inusual. Si además se dispone de pruebas de imagen recientes donde esa masa no estaba presente, se puede afirmar que la instauración de la misma ha ocurrido de forma aguda y se puede descartar otras entidades que forman parte del diagnóstico diferencial de una masa relacionada con la aurícula izquierda. La sospecha diagnóstica en el caso planteado, por tanto, se centrará en complicaciones hemorrágicas por el antecedente del intervencionismo coronario, es decir, el hematoma intramural y la disección auricular.

El hematoma intramural auricular es una entidad clínica distinta con características fisiopatológicas que difieren de la disección auricular. Sin embargo, existe una superposición en la literatura entre las dos condiciones. Ambas patologías son entidades poco frecuentes y la información de la que se dispone se basa en casos aislados descritos en la literatura. En ambos casos la presentación clínica dependerá de la severidad y de la afectación sobre circulación cardiovascular.

Se cree que la disección auricular se genera a partir de una rotura contenida auriculoventricular, permitiendo que la sangre de una cavidad de alta presión como el ventrículo izquierdo, separe las capas de la aurícula izquierda y se forme así una luz falsa. Las causas más frecuentes son la cirugía de la válvula mitral, el traumatismo torácico no penetrante, la ruptura parietal del ventrículo izquierdo posterior al infarto del miocardio y la endocarditis infecciosa. En la ecocardiografía se observa la aurícula izquierda como una estructura de doble pared con flujo turbulento en su espacio que crea una cámara nueva, dentro o junto a la verdadera aurícula izquierda. Esta falsa cámara aparece como un área anecoica que provoca obliteración parcial de la cavidad y con flujo en su interior. El abordaje es quirúrgico y se recomienda o bien el cierre de entrada o el drenaje interno de la luz falsa.

El hematoma intramural se produce por rotura microvascular contenida de las coronarias intramurales o subepicárdicas pudiendo ocurrir espontáneamente o secundarias a un traumatismo torácico o intervención tanto quirúrgica como, menos frecuentemente, percutánea. Cuando hay sangrado intramural, la baja presión en la aurícula izquierda permite al hematoma ocupar la cavidad auricular y secundariamente generar taponamiento intrínseco similar a la disección auricular. Ecocardiográficamente se describe generalmente como una masa homogénea, sin flujo en su interior que ocupa de forma parcial o total la cavidad auricular. El diagnóstico diferencial en estos casos se plantea con masas de aspecto quístico relacionadas con la aurícula: hidatidosis, mixoma, pseudoquistes de aurícula derecha, compresión extrínseca por neoplasias, quistes hemáticos simples, así como con la disección auricular. Asimismo, hay descritos casos donde el hematoma se describe como masa de ecogenicidad mixta. El abordaje en este caso puede ser conservador o quirúrgico con drenaje del hematoma.

Ambas entidades son de difícil diagnóstico, ya que pueden simular otras patologías: la ecocardiografía transesofágica ${ }^{(2)}$ se considera el recurso diagnóstico de elección, junto con la clínica (compromiso hemodinámico) ayudan a establecer un diagnóstico de sospecha adecuado. La disponibilidad de pruebas de imagen adicionales tales como la tomografía computarizada o la resonancia magnética ${ }^{(3,4)}$ permiten una mejor caracterización anatómica y tisular, así como la valoración de estructuras vecinas y su afectación, tales como las venas pulmonares o esófago. Sin embargo, el diagnóstico definitivo se confirma solamente tras la realización de intervencionismo quirúrgico ${ }^{(5)}$.

\section{Conclusiones}

Ante clínica o signos de compromiso hemodinámico tras una intervención percutánea la ecocardiografía resulta una herramienta fundamental para filiar posibles complicaciones derivadas de la misma. En el diagnóstico diferencial el hematoma intramural es, aunque infrecuente, una posible causa de complicaciones y su diagnóstico temprano tiene un importante impacto pronóstico(6).

La disponibilidad de pruebas de imagen adicionales como la tomografía computarizada o la resonancia magnética son de utilidad para la caracterización de la masa.

\section{Ideas para recordar}

El hematoma intramural, aunque infrecuente, es una posible complicación del intervencionismo coronario percutáneo.

- Las técnicas ecocardiográficas establecen la sospecha y permiten guiar el diagnóstico de las masas auriculares junto con otras técnicas de imagen.

\section{Bibliografía}

1. Galiuto L, Fedele E, Locorotondo G, et al. Intramural Atrial Hematoma: A Rare Complication of a Common Procedure. Echocardiography 2013; E255-E257. 
2. Lestuzzi C, Nicolosi GL, Mimo R, et al. Usefulness of transesophageal echocardiography in evaluation of paracardiac neoplastic masses. Am J Cardiol. 1992; 70: 247-251

3. Álvarez J, Rubio Á, De Mora M, et al. Hematoma intramural auricular izquierdo diagnosticado por resonancia magnética nuclear. Rev Esp Cardiol 2002; 55: 872-874.

4. Lundt JT, Ehman RL, Julsrud PR, et al. Cardiac masses: assessment by MR imaging. Am J Roentgenol 1989; 152: 469-473.
5. Jothidasan A, Attaran S, Hunter D, et al. Management of a Left Atrial Intramural Hematoma After Percutaneous Intervention. Ann Thorac Surg 2014; 97: 2196-2197.

6. Franks RJ, De Souza A, Di Mario C. Left Atrial Intramural Hematoma After Percutaneous Coronary Intervention. Catheter Cardiovasc Interv 2015: 86: E150-152. 